\title{
Highly Resolved Mn K $\beta$ Emission: A Potential Probe in Laboratory for Analysis of Ligand Coordination around Mn Atoms in Gels and Solutions
}

\author{
Hisashi HaYaShi ${ }^{\dagger}$ and Mao TAKAISHI \\ Department of Chemical and Biological Sciences, Faculty of Science, Japan Women's University, 2-8-1 Mejirodai, \\ Bunkyo, Tokyo 112-8681, Japan
}

\begin{abstract}
$\mathrm{Mn} K \beta$ spectra of $\mathrm{Mn}, \mathrm{MnO}, \mathrm{MnSO}_{4} \cdot \mathrm{H}_{2} \mathrm{O}, \mathrm{KMnO}_{4}, 0.50 \mathrm{M} \mathrm{MnSO}_{4}$ aqueous solution, and the precipitation bands of $\mathrm{Mn}$ $\mathrm{Fe}$-based Prussian blue analogs formed in $2.4 \mathrm{wt} . \%$ agarose gel ("Gel") were measured using a laboratory-use X-ray setup with $\sim 2.6 \mathrm{eV}$ instrumental resolution, which comprises a cylindrically bent $\mathrm{Si}$ (400) crystal monochromator and a spherically bent $\mathrm{Ge}(440)$ crystal analyzer. The oxidation-state dependent shift of the $\mathrm{Mn} K \beta_{1,3}$ peak $(\sim 1 \mathrm{eV})$ was clearly observed for $\mathrm{Mn}, \mathrm{MnO}$, and $\mathrm{KMnO}_{4}$, confirming that the employed setup can acquire the key features of Mn $K \beta$ spectra. The Mn $K \beta$ spectra of $\mathrm{MnSO}_{4} \cdot \mathrm{H}_{2} \mathrm{O}$, the $0.50 \mathrm{M}$ solution, and Gel exhibited small but distinguishable differences, whereas the spectra acquired at two positions in Gel were almost the same. These results suggest that highly resolved Mn $K \beta$ spectra can be helpful for assessing ligand coordination around Mn atoms in gels and solutions.
\end{abstract}

Keywords X-ray fluorescence, laboratory-use X-ray setup, spectroscopy, chemical effects, ligand coordination, precipitation band formation, gel, solution, Mn $K \beta$ emission

(Received March 10, 2020; Accepted May 1, 2020; Advance Publication Released Online by J-STAGE May 8, 2020)

\section{Introduction}

High-resolution X-ray fluorescence (HR-XRF) spectroscopy, also called X-ray emission spectroscopy, ${ }^{2,3}$ is a bulk-sensitive and non-destructive method for assessing spin and ligand characteristics of a given $3 \mathrm{~d}^{-3-13}$ or $4 \mathrm{f}-\mathrm{element}^{14-16}$ in the studied samples. HR-XRF spectroscopy, as well as its complementary technique, X-ray absorption fine structure (XAFS) spectroscopy, is advantageous in that no special sample preparation is usually required and it is applicable to crystalline materials as well as solutions, gels, or amorphous materials.

Although HR-XRF spectroscopy has a long research history of use for observing chemical effects in the laboratory, ${ }^{17-19}$ most applications of HR-XRF spectroscopy in the last 20 years (with rare exceptions, such as radiative Auger effects ${ }^{20}$ ) have been performed at synchrotron radiation (SR) facilities, which can provide bright, coherent, energy-tunable and monochromatic $\mathrm{X}$-rays as well as sophisticated detection systems. ${ }^{21}$ Excessive reliance on SR, however, hinders the application of HR-XRF spectroscopy (1) in more introductory, routine, high-throughput, or lengthy experiments and (2) for complicated, fragile, or unsafe (e.g., radioactive) samples. To make matters worse, at present, only a handful of SR stations are routinely available for HR-XRF measurements. Hence, HR-XRF spectroscopy is currently far less familiar to many analytical scientists compared to other common spectroscopic techniques such as IR, Raman, and UV-Vis spectroscopies.

Interestingly, interest in HR-XRF measurements in the

$\dagger$ To whom correspondence should be addressed.

E-mail: hayashih@fc.jwu.ac.jp laboratory is currently seeing a resurgence..$^{21-24}$ For example, very recently, highly resolved, high quality $K \beta(3 p \rightarrow 1 \mathrm{~s})$ spectra of $\mathrm{V}^{23} \mathrm{Fe},{ }^{24} \mathrm{Ni},{ }^{23,24} \mathrm{Co},{ }^{21,22} \mathrm{Cu},{ }^{24}$ and, $\mathrm{Zn}^{23}$ in several materials have been measured by different laboratory spectrometers. The spread of these "new-generation" HR-XRF spectrometers may relieve the aforementioned issues related to an excessive reliance on SR, and the resultant enhancement of the versatility of HR-XRF spectroscopy may be beneficial for analytical sciences. Meanwhile, laboratory HR-XRF applications to two chemically important systems, gels and solutions, are still scarce. Thus, in this study, we examined the applicability of HR-XRF spectroscopy for analyzing gel and solution samples. As a test HR-XRF probe, Mn $K \beta$ emission was selected, primarily because this emission has been particularly extensively used in determining the spin state, oxidation state, and ligand specification of many solid samples, including oxygen-evolving complexes,${ }^{8}$ biomimetic complexes, ${ }^{13}$ and magnetic materials. ${ }^{9,10,12}$ Furthermore, the selection of $\mathrm{Mn}$ $K \beta$ emission is also justified by the following. Recently, MnFe-based Prussian blue analogs (Mn-Fe PBAs) have been found to show several interesting crystallization/precipitation phenomena in gels, ${ }^{25-29}$ and their local structure around $\mathrm{Mn}$ atoms (examined by XAFS spectroscopy at SR facilities) has become the subject of intense research interest. ${ }^{25-27,30}$ Meanwhile, the local structure during the development of $\mathrm{Mn}-\mathrm{Fe}$ PBA precipitation bands (which can significantly influence the quality of $\mathrm{Mn}-\mathrm{Fe}$ PBAs finally formed ${ }^{31}$ ) has not been explored yet, because the time required for their full development ( $\sim 10$ days) is too long to continuously monitor their XAFS at SR facilities. Laboratory Mn $K \beta$ spectroscopy is expected to be potentially useful also for in-situ observation of the Mn local structure during the development of the Mn-Fe PBA bands in gels. 


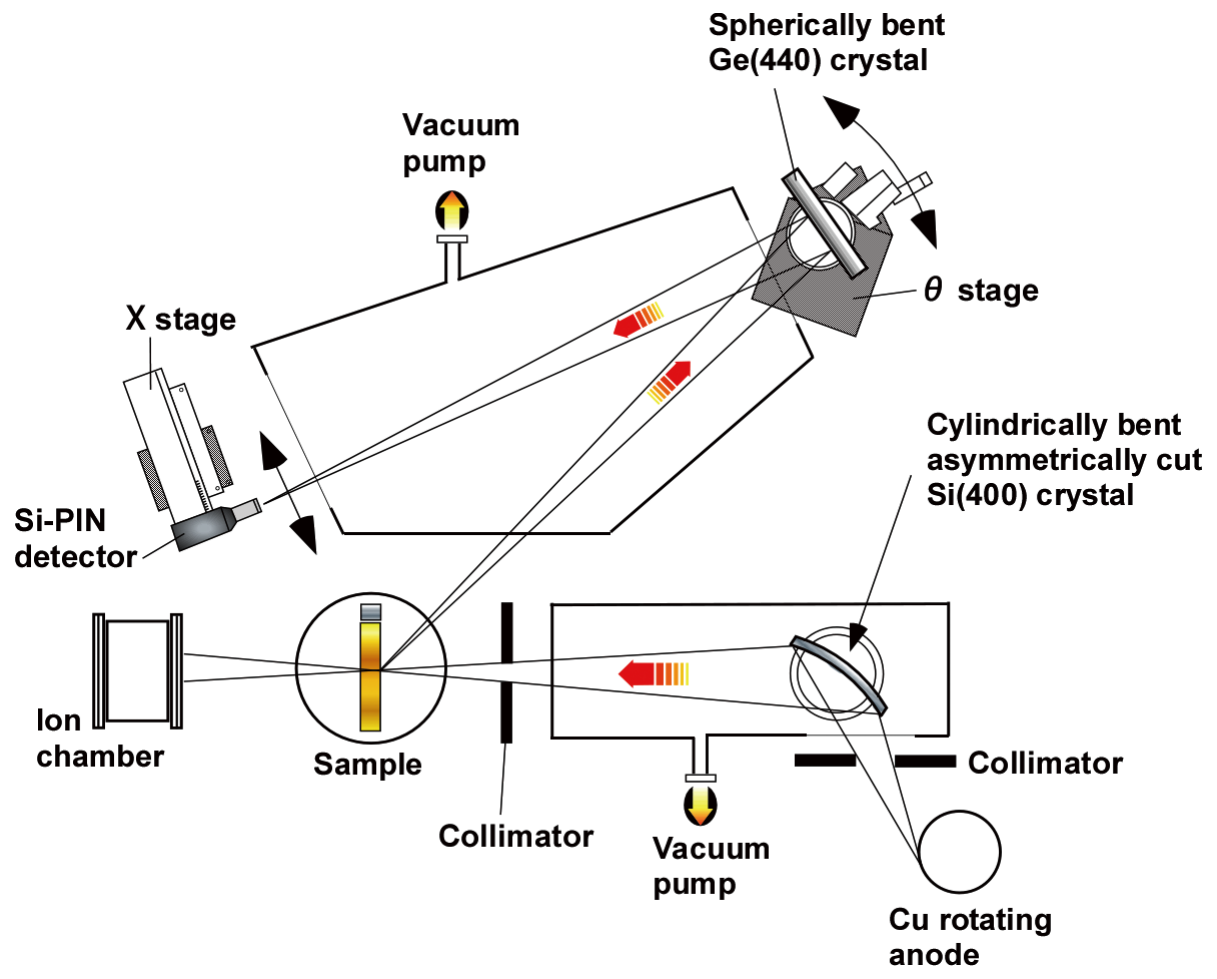

Fig. 1 Schematic representation of a laboratory-use setup to measure highly resolved Mn $K \beta$ spectra.

\section{Experimental}

\section{Chemicals}

Analytical reagent-grade powders of $\mathrm{Mn}, \mathrm{MnO}, \mathrm{MnSO}_{4} \cdot \mathrm{H}_{2} \mathrm{O}$, $\mathrm{KMnO}_{4}$, and $\mathrm{K}_{3}\left[\mathrm{Fe}(\mathrm{CN})_{6}\right]$ were obtained from Wako Pure Chemical Industries (Osaka, Japan). Agarose for electrophoresis (gel strength: $1800-2300 \mathrm{~g} / \mathrm{cm}^{3}$ ) was purchased from Kanto Chemical (Tokyo, Japan). All chemicals were used without further purification. All aqueous solutions were prepared using deionized water, which was purified from tap water by a cartridge water purifier (G-10, Organo, Tokyo, Japan).

\section{Preparation of samples}

Powders of $\mathrm{Mn}, \mathrm{MnO}, \mathrm{MnSO}_{4} \cdot \mathrm{H}_{2} \mathrm{O}$, and $\mathrm{KMnO}_{4}$, as well as $0.50 \mathrm{M} \mathrm{MnSO}_{4}$ solution, were packed in sample cells prepared from plastic straws (straw cells) ${ }^{32}$ for their Mn $K \beta$ emission measurements.

A sample, named "Gel", was prepared in accordance with a previous study ${ }^{29}$ as follows. In this sample, two different electrolytes were loaded in separate gel columns in a straw cell. The longer gel column $(\sim 30 \mathrm{~mm})$ was placed at the bottom, where the periodic precipitation bands subsequently form. The shorter column $(\sim 20 \mathrm{~mm})$, on top, had a higher electrolyte concentration. To prepare the longer gel column, an appropriate amount of agarose was dissolved at $\sim 98^{\circ} \mathrm{C}$ in deionized water $(30 \mathrm{~mL})$ with vigorous stirring to prepare a sol with $2.4 \mathrm{wt} . \%$. A predetermined amount of $\mathrm{K}_{3}\left[\mathrm{Fe}(\mathrm{CN})_{6}\right]$ was added to the sol, and the mixture was stirred continuously for $30 \mathrm{~s}$ to prepare the $0.10 \mathrm{M}\left[\mathrm{Fe}(\mathrm{CN})_{6}\right]^{3-}$ sol. This sol was transferred to a straw cell using a Pasteur pipette. The hot sol was allowed to cool to $25^{\circ} \mathrm{C}$, and a solidified gel was formed within $20 \mathrm{~min}$. Moreover, to prepare the shorter column, an appropriate amount of agarose was dissolved at $\sim 98^{\circ} \mathrm{C}$ in deionized water $(30 \mathrm{~mL})$ with vigorous stirring to prepare a sol with $0.5 \mathrm{wt} . \%$. A predetermined amount of $\mathrm{MnSO}_{4} \cdot \mathrm{H}_{2} \mathrm{O}$ was added to the agarose sol, and the mixture was stirred continuously for $30 \mathrm{~s}$ to prepare the $0.50 \mathrm{M}$ $\mathrm{Mn}^{2+}$ sol. The resultant $\mathrm{Mn}^{2+}$ sol was poured over the longer $\left[\mathrm{Fe}(\mathrm{CN})_{6}\right]^{3-}$ gel column using a Pasteur pipette, and the $\mathrm{Mn}^{2+}$ sol also solidified into a gel within $20 \mathrm{~min}$. The straw cell was subsequently closed using styrene-resin stoppers covered by Teflon and was allowed to stand at $25^{\circ} \mathrm{C}$ for $\sim 8$ days to allow precipitation bands to form in the gels. The unreacted electrolytes in Gel were then removed by suction and the addition of deionized water ( $\sim 40 \mathrm{~mm}$ in height) to the longer gel column using a Pasteur pipette. This removal procedure was repeated three times every 2 days.

\section{Apparatus and measurements}

To measure highly resolved Mn $K \beta$ emission spectra, a wavelength-dispersive, laboratory-use HR-XRF setup was arranged (refer to Fig. 1). A previously reported setup s2,33 $^{32}$ was modified to form the setup for this study. The $\mathrm{Cu} K \alpha_{1}$ X-rays from an $18 \mathrm{~kW}$ generator (RU-300, Rigaku, Tokyo, Japan), operated at $40 \mathrm{kV}$ and $150 \mathrm{~mA}$, were focused within $0.5 \mathrm{~mm}$ in the horizontal direction by a $\mathrm{Si}$ (400) Johansson-type crystal monochromator (Saint-Gobain Crystals, Nemours, France). This crystal was asymmetrically cut and had a radius of curvature of $450 \mathrm{~mm}$. The focal lengths for $\mathrm{Cu} K \alpha_{1}$ were $187 \mathrm{~mm}$ (generator-monochromator) and $316 \mathrm{~mm}$ (monochromatorsample). The monochromator was aligned to obtain the maximal output current from an ion chamber (S-1194B1, Ohyo Koken Kogyo, Tokyo, Japan). A slit (Cross slit screen 3013, Huber, Rimsting, Germany) of $5 \times 7 \mathrm{~mm}^{2}$ (width height) was placed nearly $100 \mathrm{~mm}$ in front of the sample to guard against parasitic emissions from the monochromator. Before and after each $\mathrm{Mn}$ $K \beta$ measurement, the intensity of the incident $\mathrm{X}$-rays was monitored by the ion chamber, with the variation being within $2 \%$. The straw cell containing samples was mounted vertical to the line-shape X-ray beams on the focal position. No automatic 
positioning stages for the sample are currently available for this setup, so the irradiation position was changed manually.

In most laboratory-use HR-XRF spectrometers developed recently, ${ }^{21-24} \mathrm{X}$-rays from the generator are directly irradiated onto the sample to avoid the loss of the incident X-ray intensity upon introducing a monochromator. In fact, the intensity of the

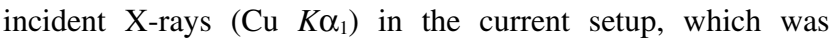
estimated by the output from the ion chamber $\left(\sim 5 \times 10^{9} / \mathrm{s}\right),{ }^{33}$ is considerably lower than the intensities of modern HR-XRF spectrometers (e.g., $\left.\sim 8 \times 10^{11} / \mathrm{s}\right) .{ }^{21} \quad$ Meanwhile, the crystal monochromator is very effective in eliminating the background in the HR-XRF spectra. Such elimination compensates, at least partially, for the intensity loss of the incident X-rays (or the intensity loss of the HR-XRF signals). Note also that linefocused X-ray beams, which are produced by the curved monochromator, are preferable to examine position dependence of HR-XRF in (pseudo-) one-dimensional systems, such as Liesegang bands in gels. ${ }^{25-27,29}$ In addition, line-focused, monochromatic X-ray incidence allows us to easily incorporate the HR-XRF setup with other X-ray instruments, such as smallangle X-ray scattering (SAXS) diffractometers ${ }^{34}$ (SAXS is a standard technique to study structural properties of gels and solutions). ${ }^{35}$

Mn $K \beta$ emission from the sample was analyzed and focused by a Ge (440) spherically bent Johann-type crystal (SaintGobain Crystals; bending radius, $820 \mathrm{~mm}$; diameter, $75 \mathrm{~mm}$ ). The crystal was placed so that the detection angles were $140^{\circ}$. To achieve high-resolution, the analyzer crystal was operated close to the back-scattering geometry, covering the Bragg angles ranging from 71.62 to $72.60^{\circ}$, corresponding to an energy range of $6464-6500 \mathrm{eV}$. The instrumental resolution of this setup will be discussed later.

Highly resolved Mn $K \beta$ spectra were measured by rotating the analyzer crystal on a computer-controlled stage (RA07A-W, Kohzu Precision, Kawasaki, Japan) and linearly moving a silicon PIN detector (XR-100CR, Amptek, Bedford, MA) on an $X$ stage (XA05A-L2, Kohzu Precision) simultaneously. Signals from the detector were fed into conventional photon-counting electronics: a timing single-channel analyzer (Model 553, Ortec, Atlanta, GA) and a dual counter/timer (Model 994, Ortec). The beam path has $50 \mu \mathrm{m}$-thick Kapton windows, and it was evacuated using a rotary pump (VR16, Koki Holdings, Tokyo, Japan) to avoid the absorption and scattering of X-rays by air. In current measurements, the Mn $K \beta$ spectra were collected over the emission energy range of $6464-6500 \mathrm{eV}$ in $0.5 \mathrm{eV}$ intervals. All measurements were performed at $25^{\circ} \mathrm{C}$.

The energies of the measured $\mathrm{Mn} K \beta_{1,3}$ peaks were determined by fitting the peaks with a Voigt function using a software for data analysis and graphing, Origin 2019 (OriginLab, Northampton, MA). The fitting errors in the calculated peak energies using Origin 2019 were within $0.05 \mathrm{eV}$. The reproducibility of the $K \beta$ spectra was checked by repeating the above-mentioned measurements at least 3 times. The differences in the $K \beta_{1,3}$ peak energies obtained by the repeatedly measured spectra were within $0.06 \mathrm{eV}$. The spectra obtained for each sample were added using Origin 2019 to reduce their statistical uncertainties. A typical collection of the Mn $K \beta$ spectra took $6(\mathrm{Mn})-24 \mathrm{~h}(\mathrm{Gel})$, depending on the number of repetitions of the measurements.

In order to estimate the distribution of Mn-Fe PBAs precipitates in Gel, energy-dispersive X-ray fluorescence (EDXRF) intensity distribution of Gel was also measured using an experimental setup, the details of which are provided elsewhere. ${ }^{28}$ Briefly, $\mathrm{Cu} \mathrm{K \alpha _{1 }}$ X-rays from RU-300, operated at $40 \mathrm{kV}$ and $60 \mathrm{~mA}$, were used as the excitation source and were focused within $0.5 \mathrm{~mm}$ in the horizontal direction by an $\mathrm{SiO}_{2}(101)$ Johansson-type crystal monochromator (Saint-Gobain Crystals). Gel was placed vertically with respect to the incident X-ray beam with line-shape on a computer-controlled $X-Z$ stage (the combination of two XA05A-L2 stages). The X-ray fluorescence signals from Gel were detected using an XR-100CR detector, and the signals were collected for $90 \mathrm{~s}$ using a multichannel analyzer (MCA8000A, Amptek).

The ED-XRF intensity distribution of Gel in the $X$-direction (the $X$-dependence of the ED-XRF intensity) was monitored at $25^{\circ} \mathrm{C}$ by moving the straw cell containing Gel in the $X$-direction in $1 \mathrm{~mm}$ increments. Following the subtraction of the constant background from the ED-XRF peaks (Mn $K \alpha$ and Fe $K \alpha$ peaks), the integrated intensities of the peaks were used to determine the ED-XRF distributions: $\mathrm{Mn} K \alpha(5691-6124 \mathrm{eV})$ and $\mathrm{Fe} K \alpha$ $(6214-6647 \mathrm{eV})$. The overlap of the Mn $K \beta-\mathrm{Fe} K \alpha$ lines in the region containing both of these elements was estimated by taking the Mn $K \beta K \beta_{1,3}+K \beta_{2,5}$ ) components in the Fe $K \alpha$ line to be $11.8 \%$ of the observed $\mathrm{Mn} K \alpha\left(K \alpha_{1}+K \alpha_{2}\right)$ intensity, as described in the literature. ${ }^{36}$

\section{Results and Discussion}

The instrumental resolution of the current setup and the confirmation of the oxidation-state dependence of $M n K \beta$ emission

Figure 2(a) compares the $\mathrm{Mn} K \beta$ spectrum of $\mathrm{MnO}$ ("Lab") acquired from the laboratory setup (see Fig. 1) with the corresponding spectrum ("SPring-8") acquired from a multicrystal, von Hamos-type setup at BL47XU, SPring-8, Japan. ${ }^{37}$ Note that the two spectra were obtained under different experimental conditions (different X-ray sources, different optics, different instrumental resolutions, different analyzer crystals, and different detectors). To better overlay with the laboratory data, the SPring- 8 spectrum was additionally broadened by convolution with a Gaussian function of FWHM $=1.2 \mathrm{eV}$ using Origin 2019. The satisfactorily good agreement found in Fig. 2(a) suggests that the instrumental resolution of the current setup is (1) lower than that of the SPring-8 setup $(\sim 1.4 \mathrm{eV}$, which was estimated from the FWHM of the elastic scattering peak for $6.4 \mathrm{keV} \mathrm{X}$-rays), ${ }^{37}$ and (2) estimated to be $\sim 1.4 \mathrm{eV}+1.2 \mathrm{eV} \approx 2.6 \mathrm{eV}$. The lower resolution of the current setup is considered to be mostly due to: (1) the different width of the irradiation area on the sample $\left(\sim 0.2 \mathrm{~mm}\right.$ in SPring- $8^{37}$ versus $\sim 0.5 \mathrm{~mm}$ in the current setup) and (2) the astigmatism of the spherically bent $\mathrm{Ge}(440)$ crystal.

Figure 2(b) shows $\mathrm{Mn} K \beta$ spectra of $\mathrm{Mn}, \mathrm{MnO}$, and $\mathrm{KMnO}_{4}$ acquired from the laboratory setup. Despite a lower instrumental resolution, the shift of the $\mathrm{Mn} K \beta_{1,3}$ peak of their compounds with the oxidation state change is clearly observable, as reported in the literature..$^{1,17-19}$ The shift values from the peak energy of Mn (so-called, chemical shift) were estimated to be $+1.1 \mathrm{eV}$ $(\mathrm{MnO})$ and $-1.0 \mathrm{eV}\left(\mathrm{KMnO}_{4}\right)$ through fitting using a Voigt function. These values agree well with the values available in the literatures: $+1.1 \mathrm{eV}(\mathrm{MnO})$ and $-1.0 \mathrm{eV}\left(\mathrm{KMnO}_{4}\right) .{ }^{17,19}$ These agreements indicate that the instrumental error of the current setup is within $0.1 \mathrm{eV}$. Moreover, considering the fitting errors $(<0.05 \mathrm{eV})$ and the precision of the spectral data (i.e., the random errors, $<0.06 \mathrm{eV}$ ), we estimate the overall experimental error in the obtained $\mathrm{Mn} K \beta_{1,3}$ peak energies to be within $\sqrt{0.1^{2}+0.05^{2}+0.06^{2}} \approx 0.13 \mathrm{eV}$.

In the lower energy side of the $K \beta_{1,3}$ peak of $\mathrm{MnO}$, a relatively intense satellite peak, i.e., Mn $K \beta^{\prime}$ peak, which is split from the $K \beta_{1,3}$ peak by (3p, 3d) exchange, ${ }^{1,2}$ can be seen, whereas the $K \beta^{\prime}$ 

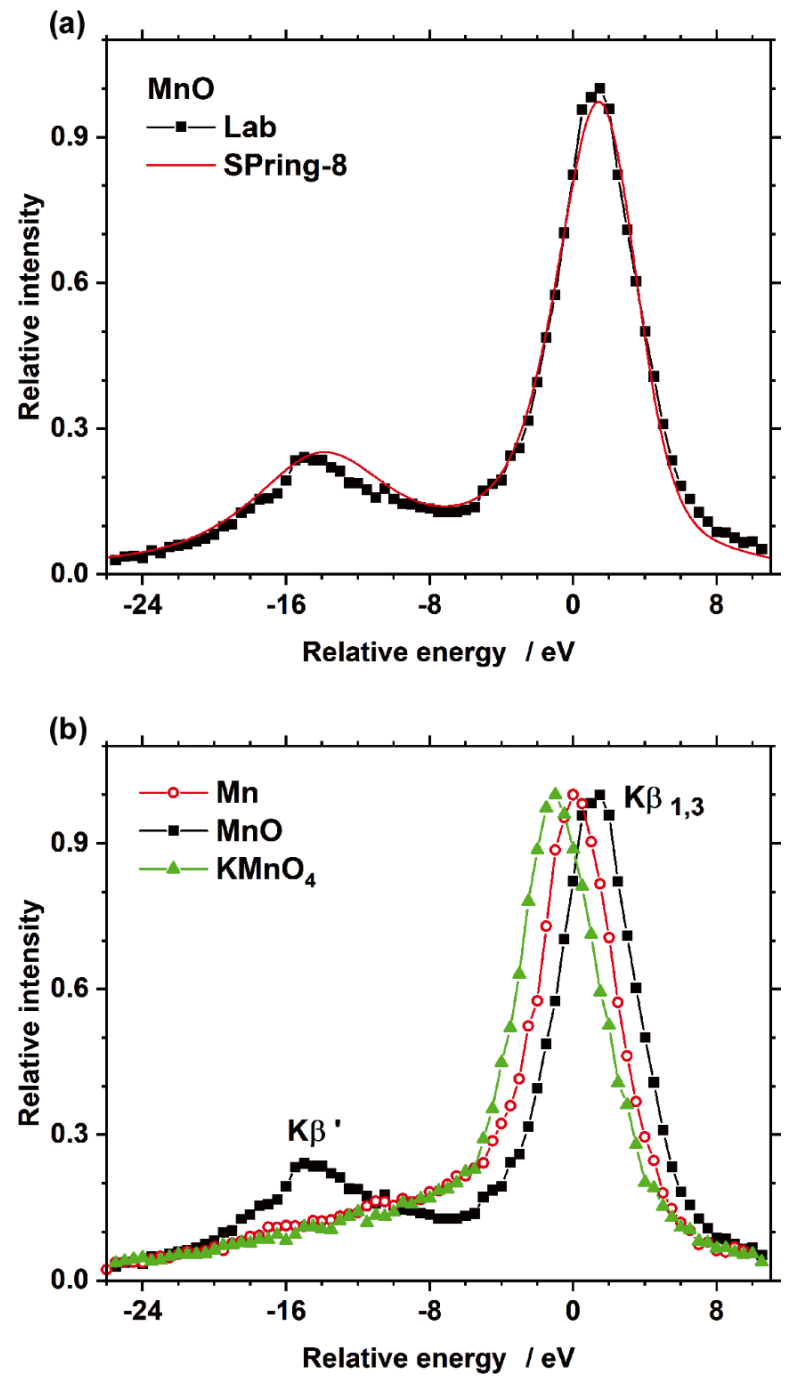

Fig. 2 (a) High-resolution $\mathrm{Mn} K \beta$ spectra of $\mathrm{MnO}$ powder. Black squares (Lab) and red line (SPring-8) show the spectrum acquired by the laboratory-use setup and the spectrum taken at SPring- 8 , respectively. (b) High-resolution $\mathrm{Mn} K \beta$ spectra of $\mathrm{Mn}, \mathrm{MnO}$, and $\mathrm{KMnO}_{4}$ powders. In both panels, the peak energy of the Mn powder is set to zero, and each peak height is normalized to one.

peak for $\mathrm{Mn}$ and $\mathrm{KMnO}_{4}$ is not very evident. These $K \beta^{\prime}$ results are also consistent with previous high-resolution $\mathrm{Mn} K \beta$ studies, ${ }^{17,18}$ including modern SR studies. ${ }^{37,38}$ Thus, Fig. 2(b) demonstrates that the instrumental resolution of the current setup $(2.6 \mathrm{eV})$, as well as the relatively small experimental error $(<0.13 \mathrm{eV})$, is sufficient to resolve the key features of Mn $K \beta$ spectra without severe loss in scientific information.

\section{Example 1: $\mathrm{Mn}^{2+}$ aqueous solution}

Figure 3 compares the $\mathrm{Mn} K \beta$ spectrum of the $0.50 \mathrm{M} \mathrm{MnSO}_{4}$ solution with the spectrum of $\mathrm{MnSO}_{4} \cdot \mathrm{H}_{2} \mathrm{O}$ powder acquired from the laboratory setup in Fig. 1. Figure 3 shows that there is a small, but measurable, shift $(\sim 0.4 \mathrm{eV})$ between the $K \beta_{1,3}$ peaks of the two samples. The local structure around $\mathrm{Mn}$ atoms in the two samples is different: the octahedral coordination of $\mathrm{O}$ atoms with a distance of $0.220 \mathrm{~nm}$ in $\mathrm{MnSO}_{4}$ aqueous solution ${ }^{39}$ differs from the distorted octahedral coordination of $\mathrm{O}$ atoms with three $\mathrm{Mn}-\mathrm{O}$ distances of $0.212,0.214$, and $0.228 \mathrm{~nm}$ in $\mathrm{MnSO}_{4} \cdot \mathrm{H}_{2} \mathrm{O}$ powder. ${ }^{40}$ Generally, the $\mathrm{Mn} K \beta_{1,3}$ peak is less

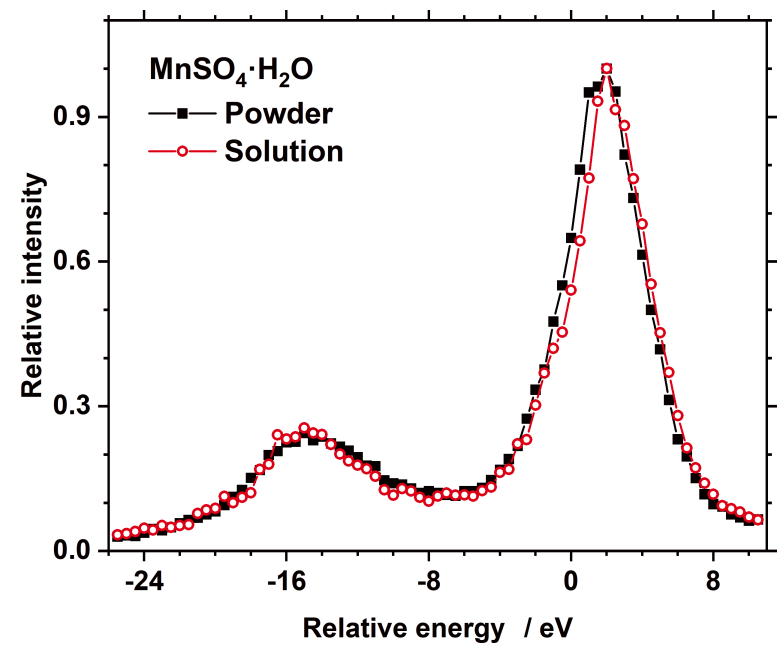

Fig. 3 High-resolution $\mathrm{Mn} K \beta$ spectra of $\mathrm{MnSO}_{4} \cdot \mathrm{H}_{2} \mathrm{O}$ powder and $0.50 \mathrm{M} \mathrm{MnSO}_{4}$ solution. The peak energy of the $\mathrm{Mn}$ powder is set to zero, and each peak height is normalized to one.

dependent on the ligand environment of $\mathrm{Mn}$ atoms than on the oxidation state. ${ }^{5,8}$ Nevertheless, the $0.4 \mathrm{eV}$ shift observed here suggests that the $K \beta_{1,3}$ peak energy is, in some cases, sensitive enough to detect changes in the ligand coordination around $\mathrm{Mn}$ atoms. The suggested $\mathrm{Mn} K \beta_{1,3}$ sensitivity is expected to be helpful for addressing issues in solution chemistry, e.g., the ligand changes of $\mathrm{Mn}$ atoms in $\mathrm{Mn}^{2+}$ aqueous solutions (including $\mathrm{MnCl}_{2}$ and $\mathrm{MnBr}_{2}$ solutions) ) $^{41,42}$ as the solute concentration increases.

\section{Example 2: Mn-Fe PBA precipitation bands formed in agarose gel}

The ED-XRF (Mn $K \alpha$ and $\mathrm{Fe} K \alpha$ ) intensity distributions of Gel obtained after 16 days from the sample preparation are shown in Fig. 4(a). The horizontal axis in Fig. 4(a) represents the distance from the gel boundary in Gel $(X)$, and its value is positive at the bottom of Gel. The vertical axis represents the relative ED-XRF intensity. These ED-XRF distributions, which provide a good approximation of the $\mathrm{Mn}$ and $\mathrm{Fe}$ distributions, are compared with the positions of the precipitation bands shown in the corresponding image at the top of the panel.

The image and ED-XRF distributions shown in Fig. 4(a) are basically similar to the results reported in previous studies. ${ }^{28-30}$ This image confirms that Mn-Fe PBAs form discrete precipitation bands in agarose gel, although their number is minimal, and their bandwidths are broad. In accordance with this observation, the ED-XRF distributions in Fig. 4(a) exhibit a rich structure. As also found in previous studies, ${ }^{25,26,28,30}$ the peak positions of the $\mathrm{Mn}$ and $\mathrm{Fe} K \alpha$ distributions coincided well with one another, suggesting that these peaks could be attributed to the presence of Mn-Fe PBAs. It is worth noting that both ED-XRF distributions fluctuated not only at the periodic band zone $(X>15 \mathrm{~mm})$, but also in the turbid zone $(0<X<15 \mathrm{~mm})$, suggesting that the Mn-Fe PBA concentration fluctuated significantly over a wide region in agarose gel, including the turbid zone.

The inset in Fig. 4(b) shows raw Mn $K \beta$ spectra (where the vertical axis represents count rates) obtained at the turbid zone $(X=3 \mathrm{~mm})$ and the periodic band zone $(X=20 \mathrm{~mm})$. These measuring points are marked by arrows in the top image in Fig. 4(a). For a comparison, the spectrum of the $0.50 \mathrm{M} \mathrm{MnSO}_{4}$ solution is also shown in the inset of Fig. $4(\mathrm{~b})$. The Mn $K \beta_{1,3}$ 

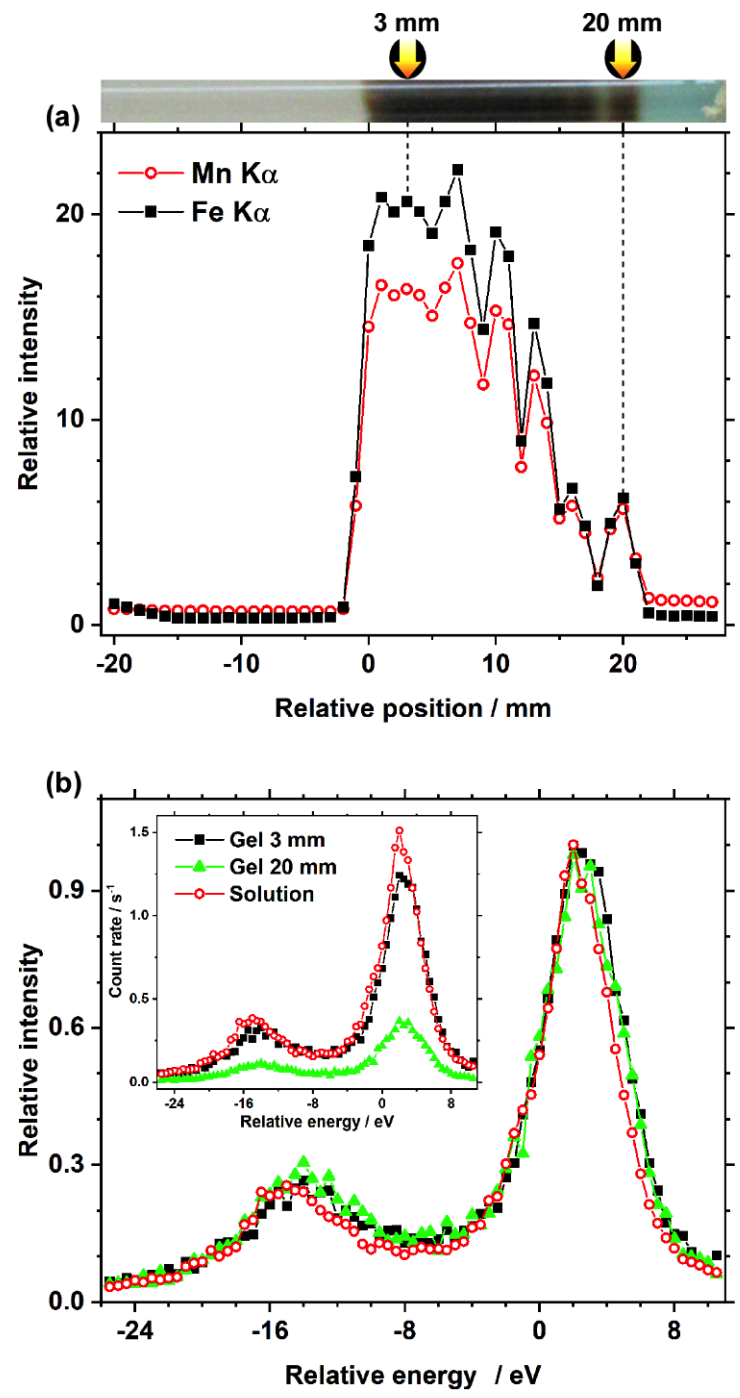

Fig. 4 (a) $\mathrm{Mn}$ and Fe $K \alpha$ intensity distribution of Gel. Captured image is displayed at the top of this figure to facilitate a comparison of the $\mathrm{Mn}$ and $\mathrm{Fe} K \alpha$ distributions with the position of the precipitation bands. The positions at which the high-resolution Mn $K \beta$ spectra (" $3 \mathrm{~mm}$ " and " $20 \mathrm{~mm}$ ") were recorded are also shown by arrows. (b) The high-resolution $\mathrm{Mn} K \beta$ spectra acquired at these positions. For comparison, the spectrum of $0.50 \mathrm{M} \mathrm{MnSO}_{4}$ solution is also shown. The peak energy of the Mn powder is set to zero. Each peak height is normalized to one, and the count rates of these samples are plotted in the inset.

peak intensity at $3 \mathrm{~mm}$ is approximately 4 -fold higher than the intensity at $20 \mathrm{~mm}$, which shows good correspondence with the ED-XRF results in Fig. 4(a). The Mn $K \beta_{1,3}$ peak intensity of the $0.50 \mathrm{M}$ solution is 1.2 -fold higher than the $3 \mathrm{~mm}$ intensity. Meanwhile, even for the $0.50 \mathrm{M}$ solution, the peak intensity is considerably low $(\sim 1.5 / \mathrm{s})$. Such weak signals might disappoint researchers expecting HR-XRF to be a potential experimental probe in laboratory. Nevertheless, the normalized Mn $K \beta$ spectra in the main panel of Fig. 4(b) could offer interesting information about Mn local structure in Gel as follows.

Comparisons of the normalized Mn $K \beta$ spectra of Gel at $X=$ 3 and $20 \mathrm{~mm}$ reveal agreement between the two spectra within experimental uncertainties (e.g., the difference of the $K \beta_{1,3}$ peak energies is $\sim 0.07<0.13 \mathrm{eV}$ ), indicating that local structures around $\mathrm{Mn}$ atoms in the $\mathrm{Mn}-\mathrm{Fe} \mathrm{PBA}$ precipitation bands are mostly position-independent.
Our previous study suggested that in the turbid zone of Mn-Fe PBAs in agarose gels, the $\mathrm{Mn}$ atoms are surrounded by one $\mathrm{O}$ atom (Mn-O length: $0.220 \mathrm{~nm}$ ) and five $\mathrm{N}$ atoms (Mn-O length: $0.221 \mathrm{~nm}$ ) with pseudo octahedral configuration. ${ }^{30}$ The good agreement of the two Gel spectra in Fig. 4(b) strongly suggests that the aforementioned local geometry around $\mathrm{Mn}$ atoms essentially holds in the periodic precipitation band zone in Gel as well. The uniformity observed in Gel (the agarose gel sample) can be useful in its future applications as radioactive Cs adsorbents, ${ }^{30}$ and interestingly, such uniformity has not observed in water-glass gel samples studied previously. ${ }^{25,26}$

Figure 4(b) also shows small but distinguishable shifts of $\mathrm{Mn}$ $K \beta$ peaks $\left(\sim 0.3 \mathrm{eV}\right.$ in $K \beta_{1,3}$ peak and $\sim 1 \mathrm{eV}$ in $K \beta^{\prime}$ peak) between $\mathrm{Gel}$ and the $0.50 \mathrm{M}$ solution. Because the bond lengths of $\mathrm{Mn}-\mathrm{N}(0.221 \mathrm{~nm})$ and $\mathrm{Mn}-\mathrm{O}(0.220 \mathrm{~nm})$ in Gel are almost the same as the $\mathrm{Mn}-\mathrm{O}$ length in the solution $(0.220 \mathrm{~nm})$, the observed difference can be attributed to the ligand difference (i.e., one $\mathrm{O}$ and five $\mathrm{N}$ atoms in Gel versus six $\mathrm{O}$ atoms in the $0.50 \mathrm{M}$ solution). Thus, Fig. 4(b) suggests that the highresolution Mn $K \beta$ spectra are applicable to determine the $\mathrm{N}$ to $\mathrm{O}$ ratio in the $\mathrm{Mn}-\mathrm{Fe}$ PBAs. This potential applicability is important, because (1) Mn-Fe PBAs have, more or less, some defects due to the absence of the $\mathrm{Fe}(\mathrm{CN})_{6}$ moiety (causing a low number of $\mathrm{N}$ atoms around $\mathrm{Mn}$ atoms) and the presence of connecting $\mathrm{H}_{2} \mathrm{O}$ molecules (causing a high number of $\mathrm{O}$ atoms around $\mathrm{Mn}$ atoms), and (2) the number of such defects (hence, the ratio of $\mathrm{N}$-to-O around the $\mathrm{Mn}$ atoms in $\mathrm{Mn}-\mathrm{Fe}$ PBAs) strongly influence their magnetic ${ }^{31}$ and electronic properties. ${ }^{43}$ Note also that the suggested relation between the Mn $K \beta$ shifts and the $\mathrm{N}$-to- $\mathrm{O}$ ratio around the $\mathrm{Mn}$ atoms provides a significant basis for future applications of high-resolution $\mathrm{Mn} K \beta$ spectroscopy to in-situ observation of the Mn states in $\mathrm{Mn}-\mathrm{Fe}$ PBA precipitation bands in gels, which was mentioned in the introduction.

\section{Conclusion}

By using a laboratory-use HR-XRF setup with $\sim 2.6 \mathrm{eV}$ instrumental resolution, comprised of a cylindrically bent monochromator and a spherically-bent analyzer, the small shifts in $\mathrm{Mn} K \beta_{1,3}$ and $\mathrm{Mn} K \beta^{\prime}$ peaks with changes in the octahedral coordination around $\mathrm{Mn}$ atoms in Gel and the $0.50 \mathrm{M} \mathrm{Mn}^{2+}$ solution are substantially measurable. Thus, the chemical effects on Mn $K \beta$ spectra of gels and solutions are measurable in the laboratory and are expected to be potentially usable for studying ligand characteristics around $\mathrm{Mn}$ atoms in the above media, thus making them useful for the defect analysis of $\mathrm{Mn}$ Fe PBAs in gels/solutions.

\section{Acknowledgements}

This study was partially supported by JSPS KAKENHI Grant Number JP19K05409.

\section{References}

1. P. Glatzel and U. Bergmann, Coord. Chem. Rev., 2005, 249, 65.

2. F. de Groot, Chem. Rev., 2001, 101, 1779.

3. A. Bhargava, C. Y. Chen, K. D. Finkelstein, M. J. Ward, and D. Robinson, Phys. Chem. Chem. Phys., 2018, 20, 28990. 
4. C. Suzuki, J. Kawai, H. Adachi, and T. Mukoyama, Chem. Phys., 1999, 247, 453.

5. G. Peng, F. M. F. de Groot, K. Hämäläinen, J. A. Moore, X. Wang, M. M. Grush, J. B. Hastings, D. P. Siddons, W. H. Armstrong, O. C. Mullins, and S. P. Cramer, J. Am. Chem. Soc., 1994, 116, 2914.

6. X. Wang, F. M. F. de Groot, and S. P. Cramer, Phys. Rev. B, 1997, 56, 4553.

7. U. Bergmann, M. M. Grush, C. R. Horne, P. DeMarois, J. E. Penner-Hahn, C. F. Yocum, D. W. Wright, C. E. Dubé, W. H. Armstrong, G. Christou, H. J. Eppley, and S. P. Cramer, J. Phys. Chem., 1998, 102, 8350.

8. H. Visser, E. Anxolabéhère-Mallart, U. Bergmann, P. Glatzel, J. H. Robblee, S. P. Cramer, J.-J. Girerd, K. Sauer, M. P. Klein, and V. K. Yachandra, J. Am. Chem. Soc., 2001, 123, 7031.

9. Q. Qian, T. A. Tyson, C.-C. Kao, M. Croft, S.-W. Cheong, G. Popov, and M. Greenblatt, Phys. Rev. B, 2001, 64, 024430 .

10. Q. Qian, T. A. Tyson, C.-C. Kao, M. Croft, and A. Yu. Ignatov, Appl. Phys. Lett., 2002, 80, 3141.

11. U. Bergmann, J. Bendix, P. Glatzel, H. B. Gray, and S. P. Cramer, J. Chem. Phys., 2002, 116, 2011.

12. H. Hayashi, A. Sato, T. Azumi, Y. Udagawa, T. Inami, K. Ishii, and K. B. Garg, Phys. Rev. B, 2006, 73, 134405.

13. S. C. Jensen, K. M. Davis, B. Sullivan, D. A. Hartzler, G. T. Seidler, D. M. Casa, E. Kasman, H. E. Colmer, A. A. Massie, T. A. Jackson, and Y. Pushkar, J. Phys. Chem. Lett., 2017, 8, 2584

14. H. Hayashi, N. Kawamura, M. Mizumaki, and T. Takabatake, Anal. Chem., 2009, 81, 1522.

15. H. Hayashi, Y. Takehara, N. Kawamura, and M. Mizumaki, Anal. Sci., 2010, 26, 885.

16. H. Hayashi, J. Electron Spectrosc. Relat. Phenom., 2014, 196, 58.

17. A. S. Koster and H. Mendel, J. Phys. Chem. Solids, 1970, 31,2511

18. K. Tsutsumi, H. Nakamori, and K. Ichikawa, Phys. Rev. B, 1976, 13, 929 .

19. H. Hayashi, "Chemical Effects in Hard X-ray Photon-In Photon-Out Spectra", in "Encyclopedia of Analytical Chemistry" 2013, John Wiley \& Sons, Chichester, DOI:19.1002/9780470027318.a9389.

20. J. Kawai, Anal. Sci., 2005, 21, 733.

21. G. T. Seidler, D. R. Mortensen, A. J. Remesnik, J. I. Pacold, N. A. Ball, N. Barry, M. Styczinski, and O. R. Hoidn, Rev. Sci. Instrum., 2014, 85, 113906

22. D. R. Mortensen, G. T. Seidler, A. S. Ditter, and P. Glatzel,
J. Phys.: Conf. Ser, 2016, 712, 012036.

23. E. P. Jahrman, W. M. Holden, A. S. Ditter, D. R. Mortensen, G. T. Seidler, T. T. Fister, S. A. Kozimor, L. F. J. Piper, J. Rana, N. C. Hyatt, and M. C. Stennett, Rev. Sci. Instrum., 2019, 90, 024106.

24. W. Błachucki, J. Czapla-Masztafiak, J. Sá, and J. Szlachetko, J. Anal. At. Spectrom., 2019, 34, 1409.

25. H. Hayashi and H. Abe, J. Anal. At. Spectrom., 2016, 31 , 1658.

26. H. Hayashi and H. Abe, Bull. Chem. Soc. Jpn., 2017, 90, 807.

27. H. Hayashi, S. Aoki, and H. Abe, ACS Omega, 2018, 3 , 4494.

28. H. Hayashi, Y. Sato, S. Aoki, and M. Takaishi, J. Anal. At Spectrom., 2019, 34, 979.

29. H. Hayashi, S. Aoki, and T. Suzuki, RSC. Adv., 2019, 9 , 36240.

30. H. Hayashi, S. Aoki, M. Takaishi, Y. Sato, and H. Abe, Phys. Chem. Chem. Phys., 2019, 21, 22553.

31. M. Verdaguer and G. Girolami, "Magnetic Prussian Blue Analogs", in "Magnetism: Molecules to Materials V", 2004, Wiley-VCH, Weinheim, Germany.

32. H. Hayashi and M. Takaishi, Anal. Sci., 2019, 35, 651.

33. H. Hayashi, Adv. X-ray Chem. Anal. Jpn., 2015, 46, 187.

34. H. Hayashi, K. Nishikawa, and T. Iijima, Jpn. J. Appl. Phys., 1989, 28, 1501.

35. P. Lindner and Th. Zemb (ed.), "Neutron, X-rays and Light: Scattering Methods Applied to Soft Condensed Matter", 2002, North-Holland, New York.

36. G. Zschornack, "Handbook of X-Ray Data", 2007, SpringerVerlag, Berlin.

37. H. Hayashi, M. Kawata, R. Takeda, Y. Udagawa, Y. Watanabe, T. Takano, S. Nanao, and N. Kawamura, J. Electron Spectrosc. Relat. Phenom., 2004, 136, 191.

38. U. Bergmann, P. Glatzel, J. H. Robblee, J. Messinger, C. Fernandez, R. Cinco, H. Visser, K. McFarlane, E. Bellacchio, S. A. Pizarro, K. Sauer, V. K. Yachandra, M. P. Klein, B. K. Cox, K. H. Nealson, and S. P. Cramer, J. Synchrotron Rad., 2001, 8, 199.

39. H. Ohtaki and T. Radnai, Chem. Rev., 1993, 93, 1157.

40. M. Wildner and G. Giester, Neues Jahrb. Mineral., Monatsh., 1991, 296.

41. Y. Tajiri, M. Ichihashi, T. Mibuchi, and H. Wakita, Bull. Chem. Soc. Jpn., 1986, 59, 1155.

42. B. Beagley, C. A. McAuliffe, S. P. B. Smith, and E. W. White, J. Phys.: Condens. Matter, 1991, 3, 7919.

43. W.-J. Li, C. Han, G. Cheng, S.-L. Chou, H.-K. Liu, and S.X. Dou, Small, 2019, 1900470. 